\section{ONOMÁVADIN}

Revista semestral de lingüística, filología y traducción
PONTIFICIA UNIVERSIDAD CATÓLICA DE CHILE CATOLICA DE CHILE
FACULTAD DE LETRAS

\title{
Pronunciación extranjera en importaciones léxicas: convencionalización puntual en español bonaerense
}

Foreign pronunciation in lexical imports: Punctual conventionalization in Buenos Aires Spanish

\section{Yolanda Hipperdinger}

Universidad Nacional del Sur

Consejo Nacional de Investigaciones Científicas y Técnicas Argentina

\section{(c) (i) $\bigodot_{\mathrm{BY}}$}

Yolanda Hipperdinger: Departamento de Humanidades, Universidad Nacional del Sur / Consejo Nacional de Investigaciones Científicas y Técnicas (CONICET), Argentina. | Correo electrónico: yhipperdinger@uns.edu.ar 
Pronunciación extranjera en importaciones léxicas: convencionalización puntual en...

\title{
Resumen
}

Nos ocupamos en este artículo del tratamiento oral de importaciones léxicas provenientes de lenguas internacionales prestigiosas en español bonaerense. Enfocamos, en particular, la extensión constatada en ese tratamiento de correspondencias grafémicofonológicas diferentes de las de la variedad lingüística receptora y miméticas respecto de las de las lenguas donantes, que por la referida extensión, y especialmente por su aplicación creativa, pueden considerarse convencionalizadas. Ofrecemos evidencia de su emancipación de las configuraciones orales completas que puedan estar convencionalizadas a su vez para importaciones léxicas particulares, sobre la base de datos obtenidos a través de la observación sistemática de empleos no inducidos y de la corroboración experimental.

Palabras clave: español bonaerense; importaciones léxicas; correspondencias grafémicofonológicas; mímesis; convencionalización puntual.

\begin{abstract}
We deal, in this article, with the oral treatment of lexical imports from prestigious international languages in Buenos Aires Spanish. We focus particularly on the extension, verified in that treatment, of graphemic-phonological correspondences which are different from those typical to the recipient linguistic variety, and mimetic regarding the donor languages; because of the referred extension, and specially because of their creative application, these correspondences can be considered conventionalized. We provide evidence of their emancipation of complete oral configurations that can be conventionalized for particular lexical imports, on the basis of data obtained through systematic observation of non-induced uses and experimental corroboration.
\end{abstract}

Keywords: Buenos Aires Spanish; lexical imports; graphemic-phonological correspondences; mimesis; punctual conventionalization. 


\section{Introducción}

El abordaje analítico del tratamiento que los hablantes de una lengua realizan de lo que reconocen como proveniente de otra puede enmarcarse en áreas diversas de las ciencias del lenguaje. En el caso del que nos ocuparemos en este artículo, la perspectiva asumida es sociolingüística y atiende básicamente a cuestiones de convencionalización ${ }^{1}$, en el marco del contacto lingüístico indirecto entre la variedad bonaerense del español de la Argentina ${ }^{2}$ y prestigiosas lenguas internacionales con rol de donantes de numerosas importaciones léxicas, cuya extranjeridad destaca en el cuadro del monolingüismo dominante en la región ${ }^{3}$.

Como en todo contacto indirecto, la prioridad en lo que atañe a los emergentes lingüísticos también en este caso corresponde a los ingresos léxicos, en particular a los que Myers-Scotton (2006: 212-213 et alibi) ha llamado culturales. Las importaciones léxicas ${ }^{4}$ en esta clase de contacto son regularmente ingresadas a través de la escritura, lo cual reviste importancia en relación con las configuraciones orales socialmente preferidas, sea que se

\footnotetext{
${ }^{1}$ Entendemos por convencionalización el consenso social sobre un determinado uso. Al respecto, véase Backus (2003: 244).

${ }^{2}$ La variedad territorial de referencia se habla en la ciudad de Buenos Aires, capital del país, la provincia homónima y buena parte de La Pampa, pero su influencia se extiende ampliamente más allá de esos límites. Por la zonificación dialectal y la denominación escogidas, así como por la aludida influencia del español bonaerense sobre otras variedades territoriales argentinas, véase principalmente Fontanella de Weinberg (2000).

3 La región abarca diversas situaciones de contacto lingüístico con lenguas indígenas, africanas e inmigratorias, en todos los casos con sustitución por el español. Sobre las políticas lingüísticas monoglósicas que impulsaron tal sustitución véase especialmente el análisis de Di Tullio (2003) sobre la "argentinización" lingüística, centrado en el de la inmigración masiva recibida por el país en el tránsito del siglo XIX al siglo XX.

${ }^{4}$ Nos referimos en lo sucesivo a importaciones léxicas eludiendo el empleo del (extendido pero controversial) término de préstamo, que suele ser usado para importaciones asimiladas —en oposición, sobre todo, a extranjerismo-; por estas distinciones y reflexiones vinculadas, véase inter alios Castillo Carballo (2006: 4; 2011: 64).
} 
Pronunciación extranjera en importaciones léxicas: convencionalización puntual en...

apliquen las correspondencias grafémico-fonológicas de la lengua receptora o que se procure imitar la realización oral de origen recuperando, para ello, otras correspondencias.

Cuando el hablante aplica en su realización oral de las importaciones léxicas la pauta de lectura de su propia lengua, ningún conocimiento adicional al de las convenciones escriturarias de la misma es requerido. En cambio, la tendencia actualmente apreciable a la imitación de la configuración oral de origen, que ha sido destacada reiteradamente en diversos entornos sobre todo para el caso de las importaciones léxicas del inglés (Miotti, 2008: 279), sí supone un conocimiento adicional: saber cómo se dice $X$ implica, como ya lo hemos señalado, recuperaciones extrañas a la lengua receptora.

Para el caso del español bonaerense, lo que nos interesa poner en foco aquí es el carácter de esas recuperaciones que, según intentaremos mostrarlo, puede caracterizarse como simplificatorio y adaptativo en el marco de la comparación interlingüística entre la lengua de origen y la receptora, y en el marco social del uso por los hablantes de esta última, como ubicado en un punto medio que exige esfuerzos diferentes para quienes conocen la lengua donante y para quienes no: para los hablantes monolingües en la lengua receptora supone aprender algo de otra, aun prescindiendo de instancias formales de transmisión y corrección, mientras que para los primeros requiere no emplear en su tratamiento de las importaciones léxicas, si quieren evitar una actuación divergente, todo lo que saben de las configuraciones orales respectivas en la lengua donante.

En este artículo nos ocuparemos, sobre la base de materiales obtenidos a través del registro de producciones orales no pautadas y de la elicitación experimental, de una serie de correspondencias de ese tipo que se abarcan en la realización oral corriente de importaciones léxicas provenientes de las lenguas internacionales de prestigio que tienen mayor presencia como donantes en la referida región dialectal: inglés, italiano, francés y alemán.

\section{El reconocimiento de la extranjeridad y el tratamiento de lo extranjero}

La primera constatación que realizamos en la línea de indagaciones avanzada, a través de la observación participante, es que los hablantes bonaerenses están familiarizados 
con la diferencia entre lo propio y lo exógeno en la escritura, que, como llevamos dicho, constituye la vía de ingreso privilegiada de las piezas léxicas importadas en el marco del predominio actual del contacto indirecto. La principal lengua donante en ese marco es (como en otras variedades de español, y en otras lenguas) el inglés, lingua franca internacional, pero el regular reconocimiento de la extranjeridad no se limita a donaciones léxicas de esa lengua: su eje no pasa por identificar la lengua donante, sino por identificar qué no es grafémicamente español.

El reconocimiento de la extranjeridad, sin embargo, no lleva mecánicamente a intentar leer las importaciones léxicas con (algún) acercamiento a la pronunciación de origen: normalmente no se constata ese intento en el caso de piezas léxicas provenientes de lenguas cuya presencia es rara en la región y/o de bajo prestigio; en cambio, las piezas léxicas importadas desde lenguas prolíficamente donantes y prestigiosas (al menos cuando se trata de importaciones más o menos recientes) por lo común no se leen empleando las correspondencias grafémico-fonológicas de la lengua receptora, o no exclusivamente. Esa distancia respecto de las pautas de la lengua que para el hablante es primaria para imitar (algo de) otra supone un esfuerzo, asentado al mismo tiempo en la disponibilidad de un modelo a imitar y en la motivación para hacerlo (que pueden entenderse como sus condiciones necesaria y suficiente, respectivamente).

Para las importaciones léxicas ingresadas y difundidas con anclaje en la escritura, la imitación de la realización oral de origen se verifica, así, como un desajuste en relación con las correspondencias puntuales entre grafía y sonido de la variedad lingüística receptora y, fundamentalmente, en el empleo de otras diferentes, del tipo de las "reglas básicas de correspondencia entre sonido y grafía en francés e inglés"5 que los hablantes "deducen" y “aplican al subsistema periférico constituido por los galicismos y anglicismos”, descriptas por Gómez Capuz (2004: 20) para el español peninsular.

\footnotetext{
${ }^{5}$ Resaltado en el original.
} 
Pronunciación extranjera en importaciones léxicas: convencionalización puntual en...

Estudios previos sobre la realización oral de importaciones léxicas en español bonaerense (incluyendo nombres propios) ${ }^{6}$, desarrollados sobre la base del registro de producciones orales no pautadas (inter alios Hipperdinger, 2010: 90-92.; Lasry, 2014a y 2014b), han mostrado para esta variedad de español la vigencia de tales correspondencias alternativas, y destacado su estabilidad en la medida en que se las constata en el habla de locutores de diferente nivel socioeducacional y familiaridad con las lenguas donantes.

En Bahía Blanca, ciudad que constituye el centro político-administrativo del sudoeste bonaerense y en la que desarrollamos nuestros estudios, la observación sistemática que realizamos de usos orales corrientes para importaciones léxicas de las lenguas donantes referidas ha revelado una extendida pronunciación que imita la de origen de forma parcial, $i$. e., que se procura mimética sin agotar las posibilidades de ese mimetismo. Así, por ejemplo, a la representación escrita (fija) hockey regularmente le corresponde una configuración oral que imita a la inglesa en la correspondencia de $h$ - con consonante, pero se ajusta a la pauta de lectura española en -ey. Otro ejemplo en relación con la misma lengua donante lo constituye berries (difundida asimismo bajo esta única forma escrita), en cuya pronunciación ordinaria coexisten la articulación española de la vibrante (múltiple) y la correspondencia de -ie con /i/. Casos como los comentados pueden comprenderse como el resultado de una combinación de lo que ya Pratt (1980), en su estudio del tratamiento de los anglicismos en español peninsular, distinguió como "pronunciación española de la grafía inglesa" e "imitación española de la pronunciación inglesa", al menos cuando tanto la forma escrita como la oral se hallan difundidas en el uso, el recurso de leer en español, accesible y aplicable a importaciones de cualesquiera lenguas, puede combinarse con una recuperación de correspondencias no españolas para generar formas que, sin ser estrictamente miméticas respecto de las de origen, se acercan a ellas. Puede considerarse,

\footnotetext{
${ }^{6}$ La obligatoriedad de su uso frente a la opcionalidad del de otras importaciones, que hace que muchas veces se los excluya del análisis, no es pertinente en este caso, en el que lo que importa es solamente la realización oral de piezas no españolas. Por la conveniencia de la inclusión de los nombres propios en indagaciones con ese objeto, véase Cabré Monné (2010: 9).

${ }^{7} \mathrm{E}$ autor las consideró asociadas de modo típico a los ingresos léxicos operados, respectivamente, por vía escrita y por vía oral, i. e., a configuraciones orales completas (v. infra).
} 
Pronunciación extranjera en importaciones léxicas: convencionalización puntual en...

en consecuencia, que estas últimas correspondencias conforman un reservorio específico para el tratamiento de las importaciones, constituyendo instancias de convencionalización puntual frente a la convencionalización de configuraciones completas para determinadas piezas léxicas.

No obstante, cabe destacar que el consenso social sobre configuraciones completas -orales o escritas - de importaciones léxicas puede enfocar tanto formas que buscan imitar las de las lenguas donantes como formas del todo adaptadas a la variedad lingüística receptora, y esto último incluso en el caso de que la configuración grafémica correspondiente se halle difundida y no haya sido modificada. Para la región dialectal bonaerense es un buen ejemplo la pronunciación generalizada del apellido del famoso actor cómico Antonio Gasalla como $/$ gasáša ${ }^{8}$, aunque en la misma región la realización regular en correspondencia con $l l$ para otros apellidos italianos es /1/, como es el caso del apellido del conocido dirigente deportivo Daniel Passarella (/pasaréla/) o del de uno de los principales animadores y productores argentinos, Marcelo Tinelli (/tinéli/). Siendo esto así, el problema principal sobre la cuestión que nos ocupa reside en cómo proceder para verificar la emancipación de correspondencias puntuales, si en el uso solo puede constatárselas en configuraciones completas.

Una primera respuesta a este interrogante puede pasar por la frecuencia con que la correspondencia aparezca implicada en configuraciones completas (estables). Por ejemplo, en nuestras observaciones sobre el particular en Bahía Blanca encontramos, para la realización oral de importaciones léxicas del italiano, que la correspondencia con una realización africada palatal es solo esporádica para $c$ ante vocal anterior y más común para $c c$ en el mismo contexto. Así, entre las designaciones de productos gastronómicos de origen italiano y consumo extendido encontramos, como configuraciones completas convencionalizadas, las de /focáča/ y /fetučíne/ ( /fetučíni/) en correspondencia con focaccia y fettuccine ( fettuccini), respectivamente. En cambio, frente a la correspondencia

\footnotetext{
${ }^{8}$ Para la transcripción fonológica nos ceñimos al esquema y las decisiones rotacionales de Fontanella de Weinberg $(1987,2000)$, autora de contribución señera al conocimiento de la variedad territorial de español sobre la que trabajamos.
} 
Pronunciación extranjera en importaciones léxicas: convencionalización puntual en...

común de aceto con /ačéto/ para el vinagre balsámico se encuentran realizaciones con /s/ para la tradicional /panséta/ (< pancetta) o la ciabatta que actualmente se ofrece en algunos locales de comida rápida. Sobre la diferencia de extensión de ambas correspondencias (destacada en Lasry, 2014a, 2014b) es ilustrativo el caso de la alternancia que constatamos, en correspondencia con la representación escrita peperoncino, entre /peperonsíno/ y /peperončíno/, siendo esta última realización el anclaje, en vistas de la mayor extensión de la correspondencia de la africada con $c c$, de la forma escrita hipercorrecta peperonccino que encontramos en Bahía Blanca en el marco de ofertas comerciales del rubro.

La evaluación de la frecuencia de las correspondencias miméticas en piezas léxicas completas, sin embargo, desconoce el hecho de que la misma escapa a la decisión del hablante particular, del cual en cambio depende la voluntad de retención de las correspondencias para que puedan servir, incluso, para piezas no generalizadas en el uso. Sobre esta base, entendemos que puede constituirse en criterio su aplicación en empleos creativos, $i$. e., su aplicación a la realización oral de palabras no españolas previamente desconocidas para el hablante. En el curso de las observaciones referidas hemos podido registrar en usos no inducidos de tal carácter entre hablantes bahienses, además de hacerlo en configuraciones orales completas, algunas correspondencias miméticas que, por lo mismo, consideramos que pueden tenerse por convencionalizadas per se. Dichas correspondencias, que nunca se alejan del inventario fonológico de la variedad lingüística receptora — por lo que la transcripción se ciñe a él一, son las siguientes ${ }^{9}$ :

A nivel vocálico:

$$
\begin{aligned}
& a-/ \mathrm{ei} /(\text { en sílaba tónica) } \\
& a+l l-/ \mathrm{o} /(\text { en posición final) } \\
& a i / a y-/ \mathrm{ei} / \\
& e-\varnothing \text { (en posición final) }
\end{aligned}
$$

\footnotetext{
${ }^{9}$ Nos limitamos a correspondencias que se muestran independientes de consideraciones morfoléxicas (como sería el caso de -tion-/šon/, o de ch-/k/ para piezas de origen grecolatino, en las importaciones del inglés). El orden que se sigue en la enumeración, en cada caso, es alfabético.
} 
$e e-/ \mathrm{i} /$

$i / y$-/ai/ (en sílaba tónica)

$i e-/ \mathrm{i} /$

$o o-/ \mathrm{u} /$

$\mathrm{ou}-\mathrm{au} / \sim \mathrm{ou}-\mathrm{u} /$

$u$-/a/ (en sílaba tónica)

$\ddot{u}$-/iu/

A nivel consonántico:

$$
\begin{aligned}
& c c i-/ \check{c} / \\
& g(+ \text { vocal anterior })-/ \check{\mathbf{s}} / \\
& h-/ \mathbf{X} / \\
& j-/ \check{\mathbf{s}} / \\
& l l-/ 1 / \\
& s c h-/ \check{\mathbf{s}} / \\
& s h-/ \check{\mathbf{s}} /^{10}
\end{aligned}
$$

Las correspondencias consignadas las registramos para importaciones de lenguas diferentes. De las que corresponden al vocalismo, las tres primeras las constatamos para el tratamiento de piezas léxicas provenientes del inglés, - - - para importaciones del inglés y del francés y las cuatro siguientes para importaciones del inglés. La alternancia que consignamos en relación con ou se debe a que verificamos su correspondencia con /au/ en importaciones del inglés y con $/ \mathrm{u} /$ en importaciones del francés. Finalmente, la correspondencia señalada entre $u$ y /a/ la constatamos para importaciones del inglés, y la de

\footnotetext{
${ }^{10}$ Respecto de /̌̌s/ en español bonaerense remitimos a las contribuciones de Fontanella de Weinberg (1979, 1987).
} 
$\ddot{u}$ con /iu/ para importaciones del alemán ${ }^{11}$. Esta última correspondencia es de especial interés, ya que como puede verse la realización oral citada recoge los rasgos de anterioridad y abocinamiento de la vocal alemana, pero desagregándolos en una secuencia de vocales españolas de su misma altura. Ello es muestra evidente de que no se trata de usos limitados a hablantes que conozcan la fonología de la lengua de origen.

Esta especificidad de algunas correspondencias según cuál sea la lengua donante, así como la transversalidad de otras, se manifiesta igualmente en las correspondencias a nivel consonántico consignadas. La primera la constatamos solamente para el tratamiento de importaciones del italiano, mientras que la segunda fue verificada para importaciones del inglés, del francés y del italiano, la tercera para importaciones del inglés y del alemán, la cuarta para importaciones del inglés y del francés, la quinta para importaciones de todas las lenguas donantes a las que atendemos y las dos últimas para importaciones del alemán y del inglés, respectivamente.

Para algunas de las correspondencias citadas encontramos además su empleo revertido sobre la escritura, cuando el productor individual parte de ellas para dar forma gráfica a importaciones léxicas cuya configuración estándar de origen no le es familiar. Un ejemplo lo constituye la grafía Burry para el nombre de pila del músico estadounidense Barry White, que encontramos en la enumeración manuscrita de sus autores musicales favoritos realizada por una hablante bonaerense de edad intermedia sin conocimientos formales de inglés ${ }^{12}$. Constituyen ejemplos, asimismo, algunas hipercorrecciones grafémicas que registramos, entre las que se cuenta la forma filletto para la importación

\footnotetext{
11 En la realización oral de al menos una importación léxica del francés, fondue, también observamos reiteradamente la misma realización como mímesis de la vocal anterior abocinada, aunque para esta lengua no la hemos registrado en empleos creativos.

${ }^{12}$ Una evidencia particularmente interesante de la extensión de la misma correspondencia se encuentra en la revista de entretenimientos Quijote (Buenos Aires, Editorial Juegos \& Co., febrero de 2015): en un crucigrama, la respuesta prevista para la consigna "Tupperware, fiambrera" es la forma apocopada de amplio uso de esa conocida firma comercial, /táper/ - que ha pasado a servir como designación de sus productos característicos-, con la configuración grafémica táper (v. 33-49)
} 
léxica del italiano filetto, relevada en la exposición de productos de algunos locales comerciales especializados en gastronomía de Bahía Blanca.

Particularmente señalable es que, como puede apreciarse y ya lo anticipamos, todas las correspondencias grafémico-fonológicas enlistadas implican una sustitución aproximativa $^{13}$ de la realización de origen por los fonemas de la variedad lingüística receptora definidos por rasgos más similares; por ejemplo, en la correspondencia $j$-/šs que se verifica en numerosas importaciones del inglés $j$ no se asocia con el fonema palatal africado sonoro de origen, sino con el fricativo de la misma posición articulatoria que le resulta más próximo en el inventario de la variedad lingüística receptora. Para dar cuenta de casos tales en otras situaciones de contacto lingüístico indirecto, diversos autores han recurrido a la noción de compromiso: también en el caso que analizamos, "el 'compromiso' fónico tiene como objetivo mantener la entidad fónica del modelo extranjero, sin violentar las pautas fonotácticas de la lengua receptora" (Gómez Capuz, 2001: 69). La ausencia de innovación en el inventario fonológico receptor a pesar de la mímesis pretendida puede verse, en consecuencia, como una clase de adaptación, por una vía diferente de la constituida por la aplicación de la pauta de lectura española.

\section{Prueba de fuego: lectura de pseudopalabras}

Volviendo sobre el problema que planteamos supra, asumimos como criterio en relación con la emancipación de las correspondencias grafémico-fonológicas miméticas que nos interesan que no solo recurran en el uso extendido de importaciones léxicas sino que puedan ser usadas creativamente en las que son desconocidas para el hablante. Como es obvio, esto último también puede ser testeado experimentalmente.

En tal sentido, para sumar evidencia sobre la convencionalización puntual de tales correspondencias con independencia de las configuraciones orales completas de cualesquiera importaciones léxicas, diseñamos una sencilla prueba: elaboramos una lista de

13 Véase al respecto la clasificación de Gusmani (1983: 138-140.), frecuentemente recuperada en investigaciones posteriores. 
palabras que incluía, junto con palabras no españolas (algunas de uso frecuente como importaciones léxicas y otras no), pseudopalabras españolas y no españolas, y pedimos su lectura a una muestra de 20 consultantes de 18 a 30 años ${ }^{14}$ (todos hablantes nativos de español bonaerense) de nivel socioeducacional medio (10 varones y 10 mujeres) con la indicación de que, si alguna de las palabras enlistadas les resultaba desconocida, las leyeran como las pronunciarían si debieran usarlas. Entre las pseudopalabras españolas se contaron grallocho, bralloño y hedraña, y entre las no españolas gralloccio, brallogno, hacelette, hanbrocke y throoween. Como puede apreciarse, en ambos casos se incluyeron grafemas o dígrafos característicos, y se crearon asimismo pares de configuraciones formalmente similares (que no se ubicaron en la lista de modo sucesivo) ${ }^{15}$.

Los resultados obtenidos ratifican, por una parte, el tratamiento diferencial de las palabras españolas y no españolas al que nos referimos al inicio de este artículo, y, por otra parte, la convencionalización per se de correspondencias grafémico-fonológicas puntuales. Omitiendo los detalles, los resultados de mayor interés obtenidos son los siguientes:

1) En todas las palabras y pseudopalabras no españolas los hablantes consultados recurrieron a correspondencias grafémico-fonológicas diferentes de las de la lengua nativa. Por ejemplo, $h$ - se correspondió con /x/ sistemáticamente tanto en hurry como en hupsead o hanbrocke, mientras que nunca recibió correlato fonológico en háldago o hedraña.

\footnotetext{
${ }^{14}$ La tendencia a las realizaciones orales miméticas se ha descripto como más generalizada entre los jóvenes en otras situaciones de contacto indirecto, en especial con el inglés (Gómez Capuz, 2001: 41-42). En el caso argentino, y dada la presencia del inglés como lengua extranjera en el currículum oficial (Peña, 2010), todos los jóvenes consultados han tenido algún acceso formal a esa lengua; no obstante, es de destacar que la imitación que registramos se restringió en todos los casos a correspondencias puntuales y no se restringió al inglés, abarcando lenguas a las que los consultantes no tuvieron acceso formal.

${ }^{15}$ La lista completa es la siguiente: mouse, hurry, hupsead, crouton, brallogno, hacellette, disgust, mousse, spaghetti, háldago, foundry, beenden, cralajette, ghiallacia, blame, blood, huppé, drug, grallocho, flute, bralloño, hanbrocke, hachette, hedraña, hethofan, jabeckla, thriller, round, hook, blind, jabegla, gralloccio, throoween.
} 
Pronunciación extranjera en importaciones léxicas: convencionalización puntual en...

2) Esas correspondencias, limitadas a lo que se reconoce como exógeno, se ligan además de modo característico a las lenguas que se presumen donantes en cada caso. En la prueba, y como ejemplo de ello, ee se correspondió con /i/ regularmente en throoween, pero no en beenden (si bien algunos hablantes, igualmente, pronunciaron /bínden/, extendiendo la correspondencia inglesa) ${ }^{16}$.

Es de notar, adicionalmente, que algunas de esas correspondencias mostraron un grado de convencionalización más alto que otras. Así, ll se correspondió siempre con /1/ cuando la forma propuesta no se consideró española, mientras que, por ejemplo, $j$ se correspondió con /̌s/ mayoritariamente en cralajette y jabeckla, pero en algunas oportunidades también con $/ \mathrm{x} /$.

Obviamente la prueba admite refinamiento y la muestra debería ampliarse, pero igualmente consideramos que lo expuesto sirve a nuestros presentes fines para ratificar la referida emancipación de correspondencias grafémico-fonológicas miméticas, al margen de que las palabras extranjeras que las impliquen tengan o no una realización oral completa más o menos consensual.

\section{El compromiso como adaptación, o el riesgo de la divergencia}

La enumeración de correspondencias que hemos ofrecido constituye una lista ampliable, ya que es posible que la continuidad de la investigación permita registrar empleos creativos de más correspondencias en el futuro. Empero, es de destacar la total ausencia de algunas de las posibles en los usos observados. Por ejemplo, piezas léxicas del alemán como Müsli o Müller pueden oírse como /miúsli/ y /miúler/, abarcando la correspondencia consignada supra $^{17}$, mientras que no se verifica nada similar respecto de $\ddot{o}$

\footnotetext{
${ }^{16}$ Por el mismo traslado ocasional de la correspondencia modelada sobre el inglés a piezas léxicas alemanas, véase Gómez Capuz (1997: 83).

${ }^{17}$ Un caso particularmente interesante al respecto es el de la extendida realización /miúnič/ para designar la ciudad alemana cuya representación grafémica en la lengua de origen (München) incluye $\ddot{u}$, a diferencia de la
} 
alemana. Como ilustración de ello, en las realizaciones del apellido del futbolista alemán Mario Götze, frecuentemente oídas en 2014 en ocasión de disputarse la Copa Mundial de Fútbol, ö se correlacionó comúnmente con /o/, según la pauta de lectura española, o con [ö] en el caso de periodistas que conocían la forma de origen ${ }^{18}$, sin verificarse una sustitución aproximativa como la descripta. Ello patentiza hasta qué punto el fenómeno bajo análisis involucra una selección social. Si se toma en cuenta este hecho junto con el de que la convencionalización admite grados desde el que se considere su piso, puede hipotetizarse que las correspondencias fonológicas con grafemas o dígrafos reconociblemente exógenos que pueden emanciparse son las que recurren en las importaciones léxicas en circulación (con configuraciones orales completas convencionalizadas de pretensión mimética) lo bastante como para habilitar la constitución de un modelo alternativo al de la lectura española, por un lado, y proveer a los hablantes la motivación suficiente para ajustarse a él, por otro. Si bien esa hipótesis aún aguarda corroboración, es de destacar que es compatible con ella la observación de que muchas de las correspondencias que apreciamos emancipadas son comunes a dos o más lenguas donantes, así como la de que la mayoría se aplica a importaciones léxicas de la lengua hipercentral (Calvet, 1999).

El último aspecto al que aludiremos aquí es el de la referida motivación, que no solamente sostiene el esfuerzo de los monolingües, sino que suele, además, obligar a quienes son bilingües con alguna de las lenguas donantes a pronunciar distinto las mismas piezas léxicas en esas lenguas y en español. Para el bilingüe, el riesgo de no hacerlo es enfrentar el consenso y actuar de modo divergente, lo cual puede afectar la inteligibilidad de la emisión de que se trate, pero, sobre todo, puede exponerlo a una categorización social negativa como "engolado", "soberbio" o "mandaparte"19. En particular en el caso de

generalizada representación adaptada al español (Múnich); como puede apreciarse, /miúnič/ constituye un cruce entre ambas.

18 Véanse los relatos disponibles en https://www.youtube.com/watch?v=rYcQm6B8eso y https://www.youtube.com/watch?v=jSlqzVYvIak, respectivamente (consulta del 15 de marzo de 2015).

${ }^{19}$ Estas calificaciones y las que siguen pertenecen a opiniones al respecto vertidas espontáneamente, que tuvimos ocasión de oír entre hablantes bahienses. 
Pronunciación extranjera en importaciones léxicas: convencionalización puntual en...

miembros de grupos profesionales ligados a otra lengua (profesores de idiomas, traductores) resulta visible ese ajuste, muchas veces bajo la forma de autocorrecciones.

Por supuesto, la divergencia también puede ser procurada si lo que se intenciona es llamar la atención sobre el virtuosismo que permite tales realizaciones al hablante. Una categorización social positiva de "conocedores", de los que "saben cómo se dice", coexiste con la negativa en términos de posibilidad en cuanto a la recepción de las formas no ajustadas a la selección comunitaria.

Según qué quiera mostrar de sí, entonces, y cuánto riesgo esté dispuesto a correr, el hablante que puede pronunciar las importaciones de una lengua según sus pautas lo hará o no. Que exista un riesgo, no obstante, es claramente un indicador más de la clase de consenso que nos ocupa.

\section{Conclusiones}

Hemos presentado en este artículo evidencia sobre la emancipación de correspondencias grafémico-fonológicas específicas al tratamiento oral de piezas léxicas importadas en español bonaerense, sobre la base de los usos no inducidos observados y de la corroboración experimental. De acuerdo con lo expuesto, el fenómeno de convencionalización puntual en el que nos centramos es, en la medida en que supone una selección social de algunas correspondencias grafémico-fonológicas no españolas de entre todas las posibles, simplificatorio: la mímesis pretendida respecto de las configuraciones orales de origen de las importaciones léxicas tiene un claro límite en relación con lo que se recupera de la lengua donante. Esa recuperación, de hecho, se manifiesta exclusivamente como un cierto desfasaje entre la grafía y la oralidad, que es además evaluable como tal solo desde la perspectiva de las pautas de la lengua receptora.

Por otra parte, en la medida en que el inventario fonológico de la variedad lingüística receptora no resulta alterado por las correspondencias referidas, dada la general operación de una sustitución aproximativa, la convencionalización puntual que nos interesa es también adaptativa, pese a su pretensión mimética: si se debe eludir la aplicación in toto de la pauta de lectura española para demostrar que se reconoce lo extranjero, distinguiéndolo 
Pronunciación extranjera en importaciones léxicas: convencionalización puntual en...

- y separándolo - de lo propio, en términos de inventario fonológico la sustitución se ocupa de proteger lo propio de la hibridización.

\section{Bibliografía citada}

BACKUS, Ad, 2003: "Can a mixed language be conventionalized alternational codeswitching?" en Yaron MATRAS y Peter BAKKER (eds.): The mixed language debate. Theoretical and empirical advances, Berlin: Mouton / de Gruyter, 237-270.

CABré Monné, Teresa, 2010: “La adaptación de préstamos en catalán” en Ciclo de Conferencias 2010 “El espacio ibérico de las lenguas”, Madrid: Instituto Cervantes.

CAlvet, Louis-Jean, 1999: Pour une écologie des langues du monde, Paris: Plon.

Castillo Carballo, María Auxiliadora, 2006: El préstamo lingüístico en la actualidad, Madrid: Liceus.

Castillo Carballo, María Auxiliadora, 2011: "Voces extranjeras: de la realidad lingüística peninsular a la americana", Itinerarios 13, 63-84.

Di Tullio, Ángela, 2003: Políticas lingüísticas e inmigración. El caso argentino, Buenos Aires: Eudeba.

Fontanella De Weinberg, María Beatriz, 1979: Dinámica social de un cambio lingüístico. La reestructuración de las palatales en el español bonaerense, México: Universidad Nacional Autónoma de México.

Fontanella De Weinberg, María Beatriz, 1987: El español bonaerense. Cuatro siglos de evolución lingüística (1580-1980), Buenos Aires: Hachette.

Fontanella De Weinberg, María Beatriz, 2000: "El español bonaerense” en María Beatriz Fontanella DE Weinberg (coord.). El español de la Argentina y sus variedades regionales, Buenos Aires: Edicial, 37-61.

Fontanella De Weinberg, María Beatriz (coord.), 2000: El español de la Argentina y sus variedades regionales, Buenos Aires: Edicial.

GÓMEZ CAPUZ, Juan, 1997: “Towards a Typological Classification of Linguistic Borrowing (Illustrated with Anglicisms in Romance Languages)", Revista Alicantina de Estudios Ingleses 10, 81-94. 
GÓMEZ CAPUZ, Juan, 2001: "Estrategias de integración fónica de los anglicismos en un corpus de español hablado: asimilación, compromiso y efectos estructurales", Estudios de Lingüística 15, 1-85.

Gómez CAPUZ, Juan, 2004: La inmigración léxica, Madrid: Arco Libros.

GuSMANI, Roberto, 1983: Saggi sull'interferenza linguistica, Firenze: Le Lettere, vol. II.

HiPPERDINGER, Yolanda, 2010: Sobre préstamos y apellidos, Bahía Blanca: EdiUNS.

LASRY, Lucía, 2014a: “CCómo pronunciamos los apellidos?”, ponencia presentada en las $I V$ Jornadas de Lengua, Literatura y Comunicación, Viedma: Universidad Nacional del Comahue.

LASRY, Lucía, 2014b: "Los apellidos italianos: pronunciaciones a la italiana y a la argentina", ponencia presentada en la XIV Settimana della Lingua Italiana nel MondoSemana de la Italianidad, Bahía Blanca: Consolato Generale d'Italia-Asociación Dante Alighieri-Universidad Nacional del Sur.

Miotti, Renzo, 2008: “La pronunciación de los anglicismos técnicos en dos lenguas de especialidad" en Carmen Navarro, Rosa Rodríguez Abella, Francesca Dalle Pezze y Renzo Miotti (eds.), Bern: Peter Lang, 273-295.

Myers-Scotton, Carol, 2006: Multiple Voices. An Introduction to Bilingualism, Oxford: Blackwell.

PeñA, Susana, 2010: La enseñanza de inglés en la escuela pública, Buenos Aires: Universidad de San Andrés.

PRATT, Chris, 1980: El anglicismo en el español peninsular contemporáneo, Madrid: Gredos. 